\title{
Construcción de sentidos y significados de la personalidad a través de la alfabetización de adultos
}

\section{Construction of personality senses and meanings through adult literacy}

\author{
Juan Carlos Malagón Pérez ${ }^{1}$ \\ Gerardo Ortiz Moncada ${ }^{2}$
}

\begin{abstract}
RESUMEN
En México, los programas de alfabetización para adultos (INEA, 2011; INEA, 2016) a menudo se remiten a sus implicaciones en la enseñanza, en el aprendizaje y en los recursos didácticos que se ponen en juego para el desarrollo de competencias de lectura y escritura; sin embargo -desde una mirada histórico cultural- los procesos de alfabetización tienen un impacto directo en el desarrollo de la personalidad y de la identidad ciudadana (MALAGÓN, 2020). En este estudio, la personalidad se comprende como la manifestación más alta de la actividad psíquica (VYGOTSKY, 1995), la cual se encuentra en constante cambio por la concatenación en un sistema dinámico de lo cognitivo, lo afectivo y lo volitivo en diversos procesos culturales (LEONTIEV, 1978; BOZHOVICH, 1976; ASMOLOV, 2002) como es la educación. El propósito de esta investigación es analizar -desde una perspectiva vygotskiana- e interpretar los sentidos y significados que se configuran en el proceso de alfabetización en el Instituto Nacional para la Educación de los Adultos (INEA) acerca de la personalidad de los educandos. Para lograr este propósito, se hace uso de la etnografía y la entrevista como recursos metodológicos que permiten recuperar las experiencias de educandos y educadores dentro de la cotidianidad
\end{abstract}

\begin{abstract}
In México, literacy programs for (INEA, 2011; INEA, 2016) adults are frequently oriented to teaching, learning, or didactics; however, -from a historical-cultural approach- literacy programs have impact on the development of personality and citizenship-identity (MALAGÓN, 2020). Thus, personality is considered as the higher expression of psychological activeness (Vygotsky, 1995), which is constantly changing due to the cognitive, affective and willing integration into a dynamic system towards different cultural processes (LEONTIEV, 1978; BOZHOVICH, 1976; ASMOLOV, 2002) such as education itself. The purpose of this research is to analyze and interpret from a Vygotskian perspective- those meanings and senses configured throughout the literacy process at National Institute for Adult Education on the personality of students. In order to achieve this purpose, ethnography and interviews are used as methodological resources, since they are useful to recover common life educative experiences of teachers and students. The theme-analysis technique (VAN MANEN, 2003) was used to interpret the information obtained about the senses and meanings of the educative agents involved. The results obtained from the thematic analysis make it possible to
\end{abstract}

1 Universidad Pedagógica Nacional, CDMX, México. ORCID: https://orcid.org/0000-0002-22917421. E-mail: jcarlos malagon@outlook.com.

2 Universidad Pedagógica Nacional, CDMX, México. ORCID: https://orcid.org/0000-0001-78156177. E-mail: gortizm@g.upn.mx. 
educativa. La interpretación de la información se realizó desde la técnica del análisis temático propuesto por Van Manen (2003) logrando la recuperación de los sentidos y significados de los agentes educativos participantes. Los resultados obtenidos del análisis temático permiten identificar el impacto que tiene la alfabetización en el desarrollo de la personalidad, gracias a su carácter comunicativo, de mediación, de representación de la realidad y de intercambio de significados.

Palabras clave: Alfabetización de adultos. Personalidad. Sentidos. Significados.

\section{RESUMO}

No México, os programas da alfabetização do adultos (INEA, 2011; INEA, 2016) geralmente se referem a suas implicações no ensino, na aprendizagem ou nos recursos educacionais envolvidos; no entanto - do um abordagem histórico-cultural -, os processos de alfabetização têm um impacto direto no desenvolvimento da personalidade e em identidade-cidadania (MALAGÓN, 2020). Neste estudo, a personalidade é entendida como a manifestação mais alta da atividade psíquica (VYGOTSKY, 1995), que muda constantemente devido à integração do cognitivo, do afetivo e do volitivo em diversos processos culturais (LEONTIEV, 1978; BOZHOVICH, 1976; ASMOLOV, 2002) tal como é a educação. O objetivo desta pesquisa é analisar e interpretar os sentidos e significados configurados no processo de alfabetização do Instituto Nacional de Educação de Adultos (INEA) sobre a personalidade dos alunos. identify the impact that literacy has on the development of the personality, due to its communicative nature, mediation, representation of reality and exchange of meanings.

Keywords: Adult literacy. Personality. Senses. Meanings.

Para atingir esse objetivo, etnografia e entrevista são utilizadas como recursos metodológicos que permitem recuperar as experiências de alunos e educadores na rotina educacional. As informações foram interpretadas utilizando a técnica de análise temática proposta por Van Manen (2003), obtendo a recuperação dos sentidos e significados dos agentes educacionais participantes. Os resultados obtidos na análise temática permitem identificar o impacto que a alfabetização tem no desenvolvimento da personalidade, devido ao seu caráter comunicativo, mediação, representação da realidade e intercâmbio de significados.

Palavras-chave: Alfabetização de adultos. Personalidade. Sentidos. Significados.

\section{Introducción}

La personalidad ha sido estudiada desde distintas disciplinas como la psicología y desde ella, se han construido distintas miradas teóricoconceptuales. Dentro de estas miradas se encuentra el psicoanálisis, el cognoscitivismo, el enfoque histórico-cultural, entre otras. Para el caso de este último enfoque, surgido de los postulados de Lev Semenovich Vygotsky, la 
personalidad es la máxima manifestación de la actividad nerviosa superior (VYGOTSKY, 1995), la cual se vincula directamente con la conciencia del sujeto y con sus distintas formas de actividad social, además de estar constituida por tres grandes esferas: cognición, afectos y volición (LEONTIEV, 1978). Estas esferas se encuentran íntimamente ligadas de manera indisoluble dentro de los distintos sistemas de actividad del sujeto, lo cual no reduce la posibilidad de conceptualizarlas desde sus particularidades. Desde esta perspectiva, la alfabetización como recurso de movilidad del lenguaje interviene en la configuración de la personalidad dado que a través de ella elaboramos, transmitimos y preservamos las diversas formas de vida cultural e histórica y sus repertorios culturales constitutivos, simbólicos y significativos que son la base del contenido que, a partir de nuestras relaciones sociales, interiorizaremos para elaborar representaciones de la realidad, dotarlas de sentido y significado y formar nuestros sistemas funcionales como la personalidad (MALAGÓN, 2020).

\subsection{La esfera cognoscitiva de la personalidad}

La esfera cognoscitiva es una elaboración compleja e integral que se constituye mediante las relaciones interfuncionales de los reflejos que componen a la conciencia que no podrían ser ni meramente reflexológicos ni meramente culturales (VYGOTSKY, 1995). En este sentido, Smirnov et al (1997) nos advierte que los distintos procesos cognoscitivos encuentran su base material en el sistema nervioso, mientras que la fuente de su contenido se encuentra en la experiencia histórico-cultural; esto permite comprender que tanto la memoria, la atención, la senso-percepción, el lenguaje, entre otros, no son procesos superiores per se, sino que encuentran diversos momentos de sofisticación y complejización conforme el sujeto se encuentra activo en su entorno y se enriquece de diversas experiencias de los más variados contextos. Estos procesos le confieren a la personalidad la capacidad primaria de autorreferirse, autorregularse, monitorearse y monitorear su entorno (NEBILYTZIN, 1983), para de manera secundaria identificar sus necesidades 
primarias (fisiológicas) y secundarias (sociales), y con ello generar los motivos como detonante de la actividad (GALPERIN, 1992a; LURIA, 1995).

\subsection{La esfera afectiva de la personalidad}

La esfera afectiva requiere primero apelar a su componente básico que es la emoción, la cual a su vez está compuesta por factores neurofisiológicos y bioquímicos, conductuales expresivos y cognitivos experienciales (MOLTÓ, 1995). Estas emociones al desarrollarse en sistemas de actividad diversos, generan un contenido significativo-subjetivo constituido mediante la internalización personalizada de la experiencia histórico-cultural. Esta experiencia objetivizada hacia otras personas, situaciones y relaciones es lo que conforma la afectividad (ASMOLOV, 2002). Para Vygotsky (2004) esta experiencia recibe el nombre de perezhivaniye, refiriendo al proceso de internalización y significación subjetiva de la emoción en situación social; esta aportación resulta fundamental para comprender que los afectos y las emociones no son abstracciones individualizadas, ni mucho menos constituyen actos de contemplación y atribución de estados internos per se, sino que son componentes y resultado de la actividad compleja integral que se da entre los procesos fisiológicos y los procesos histórico-sociales del sujeto (BOZHOVICH, 2009; ASMOLOV, 2009).

\subsection{La esfera volitiva de la personalidad}

La esfera volitiva se caracteriza primordialmente por permitir a la personalidad el logro de metas y objetivos (LEONTIEV, 2005a). Para que esto suceda, deben darse en primera instancia una situación de por lo menos dos opciones o alternativas, entre más amplio sea el espectro desde la simple saliencia de estímulos hasta planes a futuro, mayor complejización adquirirá la esfera volitiva (ZEIGÁRNIK, 1981; ASMOLOV, 2002, 2009). Estas alternativas serán sometidas a procesos de valoración de las necesidades, los motivos y las acciones requeridas, tanto por su temporalidad como por su espacialidad (marco de la situación) para poder alcanzar el objetivo propuesto (LEONTIEV, 2005a). 
Eventualmente -luego de haber realizado la elección- se deriva la consecución del plan de acción, ya sea programado y cumplido sin variaciones o ya sea ajustado por los cambios internos o externos que la situación demande. Una vez logrado el objetivo, los motivos se ajustan ya sea por el cumplimiento de dicho logro o integrándose a nuevos motivos de acción. La esfera volitiva le permite a la personalidad establecer diversos vínculos poli-motivacionales para orientarse hacia diversas acciones en distintos momentos y situaciones (LURIA,1976; LEONTIEV, 1969; SHELDON et al, 2018).

\subsection{El carácter social de la personalidad}

La integración de lo cognitivo, lo afectivo y lo volitivo requiere cambios constantes, esto hace de la personalidad un sistema dinámico-integral (GONZÁLEZ; MITJÁNS, 1999), cuya comprensión es posible a través de la relación entre las distintas categorías que la definen y los comportamientos que le consiguen. A diferencia de las corrientes psicológicas humanistas, cognitivas y conductistas, la psicología histórico-cultural no se declina por el descubrimiento de su desarrollo en lo interno o únicamente por los estímulos externos, sino que, plantea que el sujeto como personalidad es una construcción de sus relaciones sociales y que su actividad mental no se lleva a cabo de manera aislada e individual (VYGOTSKY, 1995; BOZHOVICH, 2009; ASMOLOV, 2009). Por el contrario, éstas adquieren su funcionalidad a través de la relación dialéctica entre los aspectos biológicos y los histórico-sociales, lo cual permite que la personalidad elabore, oriente y regule el comportamiento del sujeto (ORTIZ; MARTÍNEZ, 2003; MALAGÓN, 2020).

Desde el enfoque histórico-cultural, la personalidad posee una naturaleza social, así es posible estudiarla no como una esencia espiritual, sino como un producto del desarrollo histórico-cultural (BOZHOVICH, 1976). Esto significa que el sujeto como personalidad es una construcción históricocultural de sus relaciones sociales con el entorno en el que se desenvuelve, esto es, el sistema orientativo de sus acciones no encuentra sus bases en lo interno del sujeto sino en sus relaciones sociales y en el desarrollo de su sistema de actividades (RUBINSTEIN, 1963; LEONTIEV, 2005b). 
De esta manera, es posible considerar que la personalidad no es algo preexistente, sino que se gesta en su actividad (LEONTIEV, 1978). Al respecto resulta fundamental mencionar que la actividad psíquica, desde esta mirada histórico-cultural, cuenta con dos acepciones que en castellano no podrían ser traducidas literalmente dadas las distinciones morfológicas respecto al ruso: активность-деятельность (aktivnost-deyatielnost). La primera hace referencia al acto conductual observable, mientras que la segunda hace referencia a una procesualidad constante del contenido social que se subjetiviza, lo cual encuentra su base en la actividad psicofisiológica compleja (ORTIZ; CRUZ; LEURS, 2017).

\subsection{La relación entre la personalidad y la conciencia.}

Dado que una condición primaria que determina la formación de la personalidad, es el lugar que el sujeto ocupa en el sistema de las relaciones sociales y la actividad que en el mismo cumple (BOZHOVICH, 1976, 2009), es posible plantear que el enriquecimiento de las experiencias vividas determina su conciencia en tanto sujeto de acción y no sólo de percepción de la realidad (Marx, 1962).

Esta relación entre personalidad y conciencia se genera porque los modos de vida de la comunidad enriquecen los repertorios culturales que constituyen el contenido de la actividad psíquica (деятельность); además porque el sujeto desarrolla un conjunto de funciones y procesos psíquicos con los que interactúa en su entorno, lo cual complejiza y sofistica su relación con el mundo a través de la generación de los más variados sistemas de actividad (PETROVSKY, 1985); finalmente, porque el sujeto tiene un papel activo en el que se ponen en juego las interacciones entre su actividad psíquica (деятельность) у los roles sociales que desempeña a través de una serie de acciones (активность) que vinculan el mundo interno del sujeto con el mundo externo socialmente mediado (LEONTIEV, 2005a) y que le permiten al sujeto la elaboración de diversos sentidos y significados (VYGOTSKY, 1995).

Cabe destacar, como propone Leontiev (1978) y Bozhovich (1976, 2009), que el significado al entrar al terreno de la conciencia individual, no pierde su contenido objetivo, esto es, en este terreno de la conciencia individual se 
encuentra lo que el "yo" piensa, conoce, comprende y concibe sobre un determinado fenómeno o sobre una determinada situación (ASMOLOV, 2002), lo cual podría no coincidir plenamente con el significado consensuado socialmente, permitiendo la construcción del sentido como apropiación personal de significados. Leontiev (2005b) y Bozhovich (1976) argumentan que no se trata de buscar una contradicción entre lo lógico y lo psicológico, sino que esto va en función de la diferencia entre "lo general de lo único, de lo individual".

\subsection{Personalidad y educación}

Al analizar los postulados realizados por Vygotsky en la construcción de la psicología histórico-cultural, González y Mitjáns (1999) y Pasqualini y Martins (2015) mencionan que la educación comúnmente es concebida como un proceso de enseñanza-aprendizaje, lo cual es cierto en tanto existe una persona que sabe o conoce algún tema, el manejo de algún artefacto o herramienta, alguna práctica cultural, etc. y otra persona que se encuentra con la necesidad o con el motivo para aprender, adquirir o apropiarse de dicho conocimiento; sin embargo, esa acotación de educación solamente toma sentido para el análisis de su estructura. Si nos trasladamos a un análisis procesual de la educación, entonces encontramos que -en la relación enseñanza-aprendizaje- se construye un puente entre quien enseña y quien aprende, ese puente puede estar colmado de muchas condiciones, pero aquella que resulta más importante es la comunicación (JOLIBERT; SRAÏKI, 2009). Desde esta óptica, el puente debe estar constituido por un flujo de significados entre quien enseña y quien aprende, con el fin de que las necesidades y motivos de apropiación cultural emerjan y así la educación se convierta en un proceso intersubjetivo y socialmente mediado. Si lo estructural y lo procesual arriba mencionado se trasladan a un plano funcional de la educación, podremos apreciar que el proceso de enseñanza-aprendizaje permeado por la comunicación cumple una labor social fundamental: la promoción de las distintas esferas y relaciones de la personalidad (DUSAVITSKII, 2003). Esto es, el fin último de la educación es el desarrollo de la personalidad en contextos y situaciones socialmente 
enriquecidos (GONZÁLEZ; MITJÁNS, 1999), a través de un flujo de significados que permitirá el desarrollo de una actividad psíquica (деятельность) más compleja (MALAGÓN, 2020).

\subsection{Personalidad y alfabetización}

Los procesos y funciones educativas no podrían tener configuraciones, cambios ni transformaciones trascendentales sin la alfabetización como mediador para la elaboración, transmisión e ideal permanencia de las estructuras culturales, simbólicas y significativas del entorno y su interiorización personalizada. En este sentido, el lenguaje juega un papel fundamental no sólo por su función central que es la comunicación, sino que además el lenguaje es el principal mediador para los procesos de interiorización (VYGOTSKY, 1999), desempeñando funciones psicológicas complejas como son la materialización del pensamiento, la abstracción de la realidad, la regulación de la conducta, la direccionalidad de la actividad psíquica, entre otras (TSVÉTKOVA, 1977).

En este sentido, la alfabetización como proceso de sistematización y conciencia de la lengua a través de sus formas gráficas, no sólo encuentra lugar en la escuela como un proceso educativo formalizado institucionalmente sino que también tiene una dimensión fuera de ello y ésta es la que, en la cotidianidad educativa a través de la movilidad de los símbolos, los signos, los sentidos y significados construidos sobre el entorno y "fuera" de sus estructuras sistematizadas también dota de elementos a los sujetos para realizar representaciones sobre su realidad y configurar una conciencia colectiva e individual desde este espacio que Galperin (1992a) denomina conciencia lingüística.

Esta conciencia lingüística no sólo se gesta en el estudio gráfico de la lengua, sino que toma mayor relevancia para la personalidad cuando trasladamos la lectura y la escritura a formas de actividad humana más complejas; id est, cultura escrita y cultura lectora (JOLIBERT; SRAÏKI, 2009; CASSANY, 1993). De esta forma, la alfabetización permite -mediante la adquisición y el desarrollo de la cultura de lectura y de escritura de un sistema alfabético que representa la lengua 
de un determinado entorno- elaborar de la realidad circundante representaciones, conceptos, sentidos y significados, así como, una gama de conocimientos sobre ella.

Entonces el papel de la alfabetización no es dotar al sujeto de una serie de estrategias mecánicas de codificación y decodificación de textos a través de la lectura y escritura, sino posibilitar el desarrollo de habilidades y competencias lingüísticas entendidas como recursos, como operaciones mentales, como sistemas de conocimientos-saberes, y como puentes para apropiarse de las particularidades de la realidad que desea comprender. Lo anterior se gesta a través de la palabra (VYGOTSKY, 1999) de manera integral y compleja para el acceso representacional de su entorno (ORTIZ; CRUZ; LEURS, 2017), y para la elaboración y constante reformulación de sus conceptos, significados, sentidos y motivaciones que constituirán las diferencias individuales de su personalidad dentro de sus sistemas de actividad y que fortalecerán las prácticas culturales en las que el sujeto se ve involucrado (PETROVSKY, 1985).

Finalmente, debemos decir que la personalidad, como construcción constante del desarrollo histórico-cultural del sujeto, se encuentra ligada sobre todo en sus funciones afectivas estrechamente con la alfabetización y con su dimensión de conciencia lingüística toda vez que como Gramsci, citado en Freire y Macedo (1989) nos dice que ésta es un concepto y práctica social que se encuentra vinculada históricamente a las formas y modos mediante los que se configura el conocimiento y las relaciones de poder que podrían entenderse como la construcción de los modelos de civilización que son referentes del entorno en los que se desenvuelve el sujeto. Es decir, a la construcción tanto de esos aspectos que constituyen al entorno como a las posibilidades que mediante la lectura y la escritura tiene el sujeto para realizar representaciones conceptuales de su realidad y a partir de ello, elaborar un sistema de conocimientos, motivos y emociones que serán dotadas de una carga afectiva a partir de los sentidos y significados que tengan para sí mismo y que pondrá en juego en la construcción de su identidad y en el ejercicio de su ciudadanía (BOLÍVAR, 2007). 


\subsection{Alfabetización para adultos en México}

En México, los últimos años la alfabetización ha sido un tema de atención primordial, especialmente desde que la UNESCO planteó la posibilidad de reducir en América Latina el índice de analfabetismo a 4 puntos porcentuales entre el 2012 y el 2014 (INEA, 2016). La primera Campaña de Alfabetización que se implementó como estrategia en este país fue conducida por José Vasconcelos en 1921, propuso la apertura de escuelas nocturnas y misiones culturales que consistían en seleccionar a un maestro de misiones culturales que asistiera a las comunidades indígenas y centros rurales para elegir a personas que pudieran participar como maestros en sus comunidades y alfabetizar a sus coetáneos (SANTIAGO, 1973; GÁMEZ, 1993 citados en INEA 2016.). Lázaro Cárdenas durante su gestión presidencial implementó La Campaña de Alfabetización y la Campaña Nacional de Educación Popular siendo esta última su principal estrategia para abatir el analfabetismo a partir de 1937 (INEA, 2016). Por su parte, Torres Bodet implementó dos campañas, la primera, durante el periodo en que fue Secretario de Educación de 1943 a 1946 y la segunda durante los años 50 .

Esta replica de estrategias sobre las Campañas de Alfabetización continuó hasta 1980, año en el que se propone la creación del Instituto Nacional para la Educación de los Adultos (INEA) y su culminación en 1981 (INEA, 2011). Este Instituto se creó con el propósito de continuar combatiendo el analfabetismo y extender la estrategia para combatir el rezago educativo en materia de educación básica en los años posteriores. De este modo, se han gestado Campañas contra el Analfabetismo como parte de la estrategia política de los distintos procesos gubernamentales consecuentes (INEA, 2011), siendo la última, la Campaña Nacional contra el Analfabetismo y el Rezago Educativo 2014-2018. 


\section{Método}

\section{1. Pregunta de investigación}

¿Cómo se configuran los sentidos y significados en el aula en torno a la personalidad a través del proceso de alfabetización en el INEA entre Asesores Educativos y Educandos?

\subsection{Propósito general}

Analizar e interpretar los sentidos y significados en torno a la personalidad que configuran Asesores Educativos y Educandos a través del proceso de alfabetización en el INEA.

\subsection{Propósitos específicos}

Recuperar mediante registros de campo los sentidos y significados que configuran sobre la personalidad Asesores Educativos y Educandos en el Círculo de estudio durante el proceso de alfabetización en INEA.

Analizar por medio de la entrevista los sentidos y significados que configuran sobre la personalidad Asesores Educativos y Educandos en el proceso de alfabetización en INEA.

Interpretar mediante el análisis temático los sentidos y significados que configuran sobre la personalidad Asesores Educativos y Educandos en el proceso de alfabetización en INEA.

\subsection{Escenario y participantes}

Se seleccionaron tres círculos de estudio del Instituto Nacional de Educación para Adultos en el Estado de México.

Los participantes fueron 3 asesores (profesores del INEA) y 20 educandos (adultos que estudian en el INEA).

\subsection{Procedimiento}

Fase 1. Ingreso a la comunidad

Fase 2. Registros observacionales en los Círculos de Estudio

Fase 3. Entrevista

Fase 4. Análisis temático de las voces de los participantes. 


\section{Análisis Temático}

En este apartado haremos uso de la técnica análisis temático para interpretar lo compartido por los participantes. Su propósito consiste en recuperar lo significativo de las experiencias en forma de temas a partir de la observación de los fenómenos en que se construyen (VAN MANEN, 2003). Es de gran utilidad para adentrarnos desde una postura descriptiva e interpretativa a la comprensión de estos elementos significativos de dichas experiencias. En este caso específico se recuperan aportaciones teóricas acerca de la personalidad y elementos significativos de las experiencias compartidas por los participantes en torno a los cómo se configuran dentro del proceso de alfabetización en el INEA los sentidos y significados sobre la personalidad. Los elementos que se recuperan en forma de temas son:

- El desarrollo histórico del sujeto y la personalidad.

- La importancia del aprender a leer y escribir la realidad circundante.

- El impacto del estudio en el desarrollo de la personalidad.

- La relación entre la cultura, la educación, el aprendizaje y la personalidad

- El sesgo de la importancia de la educación y sus propósitos.

- La mirada desescolarizada de la alfabetización y su impacto en la personalidad.

A continuación, damos inicio a nuestro análisis en el siguiente apartado recuperando lo que nos comparte la Asesora 1 acerca de la personalidad.

\subsection{El desarrollo histórico del sujeto y la personalidad}

-La personalidad es única en cada persona y es irrepetible, no podemos ser los mismos siempre y no podemos ser iguales a otros siempre, la personalidad es ser único, no sé.-

La personalidad no es una función que siga un patrón de desarrollo que culmine en una determinada etapa de maduración que se manifieste bajo una lógica biológica general como la etapa de la niñez, la pubertad o la senectud Asmolov (2002). La psicología histórico-cultural enfatiza que es una función psicológica superior que se desarrolla y transforma constantemente en los 
sistemas de actividad en los que se desenvuelve el sujeto (LEONTIEV, 1978, 2005a; BOZHOVICH, 2009) lo que pone a la luz la importancia de recuperar en qué sentidos somos únicos y en qué sentidos somos lo mismo que los otros con respecto a nosotros mismos (VYGOTSKY, s.f. a) con unicidad y especificidad (GIMÉNEZ, 2007). Vygotsky (s.f. b;) argumenta que la actividad mental del sujeto sólo es posible a partir de la interrelación que existe entre el entorno y él. En esta interrelación, el sujeto va configurando sus pensamientos, sus emociones, sus actividades, sus representaciones de la realidad, sus cosmovisiones culturales e históricas, motivos y motivaciones (VYGOTSKY, 2004; MOLTÓ, 1995) sólo a partir de que va aprendiendo a leer las distintas representaciones de la realidad, la diversidad de vida cultural (TSVÉTKOVA, 1977) y de la interrelación compleja que se da entre los procesos biológicos de su actividad nerviosa y los procesos histórico-culturales, es decir, del metaproceso en el que se concatenan dichos procesos en él (NEBYLÍTZIN, 1983).

Desde otro punto de vista, la Asesora 2 nos comenta sobre la personalidad: La construyes a lo largo de tu vida desde que eres un niño y la puedes ir modificando, cambiando o mejorando en el transcurso de tu vida, yo lo concibo de esta forma.-

La personalidad como función psicológica superior integral y compleja, es un proceso que se verá siempre enriquecido por las relaciones dialéctico-materialistas que el sujeto lleva a cabo durante su desarrollo histórico-cultural (BOZHOVICH, 1976; GONZÁLEZ, 1985) y por los recursos significativos y simbólicos que se construyen en los espacios de interacción familiares, sociales y educativos (GONZÁLEZ; MITJÁNS, 1999; GALPERIN, 1992b) en los que se desenvuelve. El lenguaje juega un papel fundamental dado que es un elemento que se encuentra en todo momento presente en el desarrollo del sujeto (VYGOTSKY, 1995), empero, debemos puntualizar que, en un primer momento, los vínculos que establece en la infancia con la madre son directos y emocionales, posteriormente, cada uno de ellos se encuentra representado por el lenguaje o, mejor dicho, a partir de que le va siendo posible poner en juego por sí mismo el lenguaje. Esto le permite enriquecer su experiencia, desarrollar nuevas formas de comportamiento y nuevas formas de organización de su actividad mental (LURIA, 1984; ASMOLOV, 2009). 
Desde el inicio del desarrollo podemos ver que se ponen en juego procesos de alfabetización en los ambientes familiares y extraescolares (TEBEROSKY; SOLER, 2003) pues los procesos de lectura y escritura de la realidad que ejecuta la madre son el punto de partida para compartir la experiencia humana al sujeto en sus primeras etapas del desarrollo y en el ulterior. Es mediante este proceso que se comienzan a elaborar las bases de su actividad mental y las de los procesos histórico-sociales que le permitirán comenzar a interactuar con el entorno (VYGOTSKY, s.f. a; ASMOLOV, 2002), comprender su realidad circundante, enriquecer su experiencia y elaborar sus motivos y motivaciones (GONZÁLEZ; MITJÁNS, 1999).

\subsection{La importancia del aprender a leer y escribir la realidad circundante}

La Asesora 1 enfatizaba el valor del conocimiento al hablar del por qué considera que es importante aprender a leer y escribir en el siguiente comentario: -Es una parte fundamental porque de ahi se desprenden muchas cosas para desarrollarse como persona. Tomo mucho de ejemplo a la educando porque ahora ve otras cosas, lee más y a sus 50 años tal vez ya considera que es muy importante preguntar y preguntarse. El hecho de aprender a leer y comprender lo que está leyendo hace que su necesidad de conocimiento sea mayor, por ejemplo, quiere saber del universo, quiere saber de los planetas y de cosas que a lo mejor no tenía tan enfocadas o tan conocidas.-

González y Mitjáns (1999) y Rubinstein (1963) declaran que existe una relación indisoluble y necesaria entre los conocimientos y el desarrollo de la personalidad. Este conocimiento que puede ser comprendido como la experiencia humana que se ha construido de la interacción histórica del sujeto con el entorno (VYGOTSKY, 1995; LURIA, 1984, 1976) se pone en juego en las relaciones activas y de comunicación que establece con el mismo y con sus coetáneos (PASQUALINI; MARTINS, 2015). En dichas relaciones se dan procesos de enseñanza-aprendizaje que tienen impacto en el desarrollo de la personalidad y en los que se hace fundamental la participación activa tanto de quienes enseñan como de quienes aprenden. 
Tales procesos que se han estandarizado bajo una lógica tecnicista perfeccionista y mecanicista (BOURDIEU; PASSERON, 1996; GONZÁLEZ; MITJÁNS, 1999) que delinea ciertos roles separados en el proceso de enseñanza del proceso de aprendizaje, son procesos que desde la perspectiva de la psicología histórico-cultural se conectan en el guión que representa este puente de comunicación profunda de sentidos y significados de las individualidades cotidianas, sociales, culturales e históricas de educadores y educandos, de padres de familia e hijos etc. (MALAGÓN, 2020) esto nos permite ver que el desarrollo psicológico y el de la personalidad, no sería posible sin estos procesos complejos de actividad y de relaciones sociales (ORTIZ; CRUZ; LEURS, 2017). Por consiguiente, Luria (1984) plantea que no sólo resulta fundamental definir la naturaleza de las funciones psicológicas superiores sino también seguir meticulosamente los procesos complejos mediante los cuales el sujeto a partir de la construcción de sus relaciones sociales a través de la palabra, va formando comportamientos complejos.

\subsection{El impacto del estudio en el desarrollo de la personalidad}

La Asesora 2 comparte lo siguiente acerca del impacto que tiene en la personalidad de los Educandos el estudiar en INEA: -Se conciben como personas capaces de poder expresarse y como alguien que ya no va a tener miedo de hablar o de acercarse con otra persona porque asumen que pueden expresar su opinión y conocen aspectos que antes no sabian o por lo menos no tenían acceso a ello.-

Por su parte, la Educando 2 comenta lo siguiente acerca de si ha notado algún cambio en ella antes de estudiar y después de estudiar: -Ahora que volví a retomar mis estudios me siento diferente porque me he dado cuenta que soy capaz de hacer muchas cosas, soy capaz de desenvolverme más ante la gente, cuando luego platico con las personas de repente como que no entendía las cosas que me estaban explicando y ahorita ya con mi hija que está estudiando la preparatoria le entiendo más a lo que me explica y a sus trabajos.-

Luria (1984) enfatiza sobre la actividad mental lo siguiente: "El niño, al hacer suyas todas las técnicas principales de relación que le proponen los adultos, llega a ser capaz de modificar activamente el medio que actúa sobre él; 
el niño, al utilizar el lenguaje para su uso personal, altera la fuerza correspondiente de los estímulos que actúan sobre él y adapta su comportamiento a las influencias así modificadas." (p. 16)

En cuanto al impacto de la alfabetización de adultos en el desarrollo de la personalidad, tendríamos que preguntarnos ¿Qué tan desarrollado se encuentra este principio de la actividad mental del que nos habla Luria cuando existe una tendencia mayor a repetir y reproducir ciertas pautas de comportamiento en la vida adulta? González y Mitjans (1999) comentan que existen dos niveles de regulación de la personalidad: a) el nivel de normas, estereotipos y valores y; b) el nivel consciente volitivo. Con respecto al primero, arguyen que el sujeto da poca movilidad a la información que le llega del entorno en el que se desenvuelve porque durante su desarrollo histórico fue aprendiendo a hacerse más consciente de esos patrones que le enseñaron a reproducir que de aquello que va sucediendo en el entorno y que es posible mirar desde otras perspectivas históricas y culturales. Sobre el nivel consciente-volitivo mencionan que el sujeto da mayor movilidad a la información proveniente del entorno y a su sistema de conocimientos, resignificando, a través de un proceso de evaluación, su comportamiento y su sistema de actividades en función de esas otras perspectivas de la cuales se hace consciente a partir de que es capaz de insertarse en los fenómenos en los que se va suscitando la cotidianidad.

La cuestión es, si aquel principio de la actividad mental del que nos habla Luria (1984) se encuentra oculto en la consciencia de reproducir pautas de comportamiento, entonces la configuración de la personalidad tendrá poca movilidad, siempre estará en constante desarrollo, pero limitada a esta forma de consciencia. Por lo tanto, la alfabetización debe ser un proceso que nos permita leer, adentrarnos a y comprender esas distintas miradas que se construyen sobre la realidad circundante (FREIRE; MACEDO 1989; FERREIRO, 2002) para reorientar de manera dinámica nuestro sistema comportamental y nuestro sistema de actividad (LEONTIEV, 1969; GONZÁLEZ 1985). 


\subsection{La relación entre la cultura, la educación, el aprendizaje y la personalidad}

En otro orden de ideas, la Asesora 4 comenta lo siguiente sobre la personalidad: -Yo llamo que sería una evolución de pensamiento porque ya no soy la misma de aquellos tiempos. Tengo nuevas cosas, complemento lo que aprendí con lo que está en mi entorno.-

Desde esta perspectiva de la psicología dialéctica debemos enfatizar que referirnos a la evolución del pensamiento es comprender que su formación, el lenguaje, la actividad mental, la elaboración de un sistema de conocimientos, la posibilidad de apropiarse de la realidad a través de la elaboración de representaciones, configurar motivos y motivaciones, así como orientar su sistema comportamental, se encuentra en la relación metaprocesual que emerge de la integración compleja de las funciones del sistema nervioso y del desarrollo histórico-cultural del sujeto, lo que posibilita la construcción de una vida activa social, de una vida cultural y de una vida histórica (VYGOTSKY, s.f. b; SMIRNOV et. al, 1997; PASQUALINI; MARTINS, 2015).

Por otro lado, la Asesora 2 comentó lo siguiente con relación a cuáles considera que son las características de la personalidad: -Creencias, carácter, preferencias, educación, familia, creo que sería eso. Pienso que se manifiestan en la forma en que habla y cómo se expresa el sujeto.-

Asimismo, la Asesora 4 comentó: -La cultura, el aprendizaje, lo que te rodea, digamos el ámbito familiar, el ámbito pues hasta político, para mí la personalidad es muy importante para poder desarrollarme. Todo lo que tenemos alrededor nos complementa, bueno, siento que asi es, que todos nos complementamos.-

Ambas participaciones nos invitan a reflexionar acerca de la diferencia entre la noción de individualidad en el sujeto y la noción de lo social en el sujeto. Rubinstein (1963) plantea que un sujeto es individualidad dado que posee un conjunto de aptitudes, características y mecanismos biológicos que le son únicos e irrepetibles y social toda vez que es personalidad, esto es, de manera consciente determina su actitud hacia lo que le rodea, en la medida en que es activo con respecto a sus sistemas de actividad y a sus relaciones sociales y cada 
vez menos neutral, indiferente y pasivo con respecto a lo que se encuentra y sucede en el entorno en el que se desenvuelve. Su actividad mental y sus funciones psicológicas superiores no podrían desarrollarse sin la alfabetización como mediador para la elaboración de las cosmovisiones culturales, sus repertorios constitutivos, sus estructuras simbólicas y significativas y como proceso complejo de formación de una serie de recursos psicológicos que le permitan comprender aquello que sucede en el entorno en el que se desenvuelve desde distintas perspectivas, transmitir sus riquezas culturales, conocimientos e historia y transformar, a partir de un ejercicio de resignificación mediante la lectura de su realidad circundante, las distintas esferas de su entorno participando activamente (CASSANY, 1993; JOLIBERT; SRAÏKI, 2009).

\subsection{El sesgo de la importancia y los propósitos de la educación}

La Educando 4 comparte lo siguiente sobre la importancia de aprender a leer y escribir: -Pienso que es lo mejor. Mis papás cuando era pequeña nunca me quisieron mandar a la escuela decían que las mujeres no tenemos derecho a los estudios más que a trabajar, nada más. Desde que tengo uso de razón, ¿qué será?, desde los tres, cuatro años, andaba en la calle vendiendo paletas de hielo, vendiendo dulces, lavando trastes, o sea, hacía actividades que, pues no me correspondían, yo lo que quería era estudiar y nunca tuve la oportunidad de hacerlo. Me casé y quise que mis hijos tuvieran estudios, pero tampoco lo supieron aprovechar, se quedaron todos con la secundaria. Después sentí que ya era demasiado grande para aprender, que ya para qué lo hacía, papeles ya no necesitaba porque ya no iba a emplearme en ninguna parte, sin embargo, no me había dado cuenta de que yo con mis hijos nunca participé en sus tareas, los dejé a la deriva, o sea, yo los mandaba a la escuela, los veía que estudiaban y hacían su tarea, pero yo no participaba con ellos ¿Por qué? Pues porque yo no sabía. -

La Educando 3 comenta lo siguiente sobre el por qué considera que es importante estudiar: -Porque muchas personas cuando no tienen carrera los tratan menos. A mi me ha pasado que me humillaban por medio de que no tenemos estudios.- 
Podemos ver que la educación se ha pensado ajena a la cotidianidad, como un proceso que permite más la adquisición de un "valor personal mayor al de los otros" que como un proceso que nos permite construir espacios de interacción y herramientas para comprender y transformar el entorno en el que nos desenvolvemos (SKLIAR, 2007). Estas pautas comportamentales condicionantes de las que nos hablan ambas participantes, no son una construcción de sus propias formas de pensar o de eso a lo que llaman "cada cabeza es un mundo" tales miradas son una construcción socio-histórica (BOZHOVICH, 1976; DUSAVITSKII, 2003) que se ha delineado mediante una serie de prácticas pertenecientes a un modelo de civilización con una visión de vencidos (FANÓN, 1963).

González y Mitjáns (1999) mencionan que existen dos formas de orientación del sujeto: a) orientación activa-transformadora y; b) orientación pasivo-descriptiva. Un sujeto que se encuentra en el nivel de orientación activatransformadora será partícipe de su propio proceso de aprendizaje, dicho proceso es puesto en juego en el sistema de actividades que el sujeto realiza dentro y fuera de la escuela. Al hacerse consciente de esto, es activo en cuanto a la individualización y resignificación de la información proveniente del entorno en la medida en que sus necesidades dejan de ser vinculadas a la reproducción de pautas de comportamiento. El sujeto que se encuentra en el nivel de orientación pasivo-descriptiva no es partícipe de su proceso de aprendizaje, desarrolla una forma de consciencia de reproducción de pautas comportamentales. No mira otras perspectivas implicadas en su cotidianidad y sólo pone en juego la información relacionada con esas formas de reproducción de comportamientos que ha aprendido. Pensamos que resulta fundamental construir una educación de la personalidad (BOZHOVICH, 1976; GONZÁLEZ; MITJÁNS, 1999) que asuma los procesos educativos vinculados a la cotidianidad y a sus peculiaridades fenomenológicas, ya que, es en las relaciones que se configuran tanto en los espacios escolarizados como en los familiares y sociales y en los sistemas de actividad del sujeto donde se construyen sus sistemas de orientación y regulación comportamental, la esfera afectiva de la personalidad, su sistema motivacional, su sistema de conocimientos 
individualizado y su actividad psicológica (VYGOTSKY, 2004; GONZÁLEZ, 1985). Y, en consecuencia, las bases posteriores de las relaciones que establecerá con la familia y la sociedad (ORTIZ; MARTÍNEZ, 2003).

La Asesora 4 recupera el valor de comprender el mundo en el acto de estudiar de la siguiente manera: -Para superarte, pero no solamente por el hecho de obtener un documento como tal. Esto es un bien que te va a permitir comprender el mundo que te rodea, básicamente por eso es importante estudiar, no solamente por obtener un documento sino por las habilidades que se adquieren.-

Con respecto al desarrollo de la personalidad, asumir que estas habilidades que se construyen en el proceso de estudio son constitutivas de nuestro comportamiento global, lo que nos muestra el reto que tenemos de elaborar un concepto y una práctica educativa con respecto a la alfabetización orientados hacia la lectura, escritura y comprensión de estas diversas formas de representación de la realidad y hacia la recuperación de la riqueza de experiencias, conocimientos y herramientas de las diversas formas de vida humana para transformar nuestro entorno, nuestra cotidianidad y atender las distintas necesidades diferenciadas de todos (BOLÍVAR, 2007; LÓPEZ, 2009), así como para orientar el comportamiento y la actividad desde y dentro de otras miradas históricas y culturales. (ORTIZ; MARTÍNEZ, 2003; MALAGÓN, 2020)

\subsection{La mirada desescolarizada de la alfabetización y su impacto en la personalidad}

La Asesora 4 comenta lo siguiente sobre la alfabetización: -Es muy importante para todos, aun hasta para un joven que está en la secundaria que no sabe leer (risas) y no sabe escribir. Es muy importante para poder tener mejores oportunidades, el chico que cree que salió de la secundaria y que no necesita aprender está a la deriva porque en realidad él necesita mucho, por ejemplo, el tener que relacionar su pensamiento con lo que está viviendo, esto podría verlo como de "ay, para qué nos sirve esto o aquello si no se relaciona conmigo" entonces, creo que para mí sería que es importante aprender y poner en práctica lo que ha estudiado a lo que ha vivido hasta este momento.- 
Del mismo modo, la Educando 2 comentó: - $A$ mi parecer es importante para que podamos conocer nuestros derechos y nos sepamos defender de cualquier situación de opresión, abuso y desvalorización. Muchas veces si no sabemos leer y escribir nos engañan. Por ejemplo, si no sabemos sumar, restar y todo eso, pues también nos pueden hacer fraudes porque no conocemos.-

Ferreiro (2002) y Freire (1999) refieren que el proceso de alfabetización debe posibilitar al sujeto comprender que se construyen diversas formas de representar su existencia en el mundo, así como dar lectura a esos significados que tienen estas representaciones desde esas cosmovisiones culturales y desde esas peculiaridades fenomenológicas mediante las que se fueron construyendo y en las que se desenvuelve cotidianamente.

Por su parte, la Asesora 1 comenta lo siguiente acerca de una relación entre la alfabetización y los cómo te piensas en el mundo: - Sí, yo creo que sí porque cuando ya sabes leer y escribir y ya sabes que hay otros paises, que hay otro tipo de gente, que hay tecnología, qué hay más allá de lo que tú vives es gracias a que sabes leer y sabes comprender, tienes conocimiento a través de la lectura, a través de la tecnología, a través de todo lo que está a tu alcance.-

Esto pone énfasis en que la alfabetización no sólo debe ser entendida como una forma de sistematización y consciencia de la lengua en su forma gráfica ni como un proceso sólo de la escuela. La alfabetización es también un proceso de movilidad del lenguaje (JOLIBERT; SRAÏKI, 2009). De acuerdo con Vygotsky (1995) y Pasqualini y Martins (2015) este juega un papel central tanto en los procesos de comunicación como en los procesos de individualización de información que permitirá en conjunción con las actividades funcionales del sistema nervioso, la actividad mental del sujeto, el desarrollo y orientación de su comportamiento y el ulterior desarrollo de sus funciones psicológicas superiores en los espacios en los que se desenvuelve.

\section{Discusión}

El presente trabajo consistió en analizar -desde una perspectiva descriptivo-interpretativa- y desde la psicología histórico-cultural el impacto 
que la alfabetización tiene en el desarrollo de la personalidad de educandos y educadores en el INEA. La premisa fundamental de esta corriente teórica nos dice que la actividad mental del sujeto y su desarrollo psicológico se encuentra determinado por el proceso complejo e integral que se da entre los procesos de su actividad nerviosa superior y los procesos histórico-culturales en los que el lenguaje tiene un papel fundamental.

Las vivencias compartidas por los participantes nos muestran que la alfabetización es un proceso mucho más complejo en el que se pone en juego el lenguaje y adquiere funcionalidad como principal elemento de comunicación y como mediador de la actividad psicológica para elaborar, preservar y compartir la riqueza cultural, histórica, simbólica, significativa y de conocimientos sobre el entorno. La alfabetización entonces tiene al menos dos funciones, esta que hemos mencionado y ser un proceso fundamental en el que adquiere funcionalidad el lenguaje para el desarrollo de las funciones psicológicas superiores y el de la personalidad.

\section{Conclusiones}

A manera de conclusión, la alfabetización es un proceso que se pone en juego desde el inicio del desarrollo histórico-cultural del sujeto y en las diversas vías mediante las que se ha elaborado, conservado y transmitido la riqueza cultural, simbólica y significativa de la experiencia humana. Cada uno de estos elementos histórico-culturales son parte del proceso que le permiten el desarrollo de su actividad mental por ello la alfabetización es un proceso mediador en el que los sistemas funcionales psicológicos complejos del sujeto se desarrollan. Finalmente, esta actividad mediatizada por el lenguaje, le permitirá al sujeto elaborar un sistema de conocimientos individualizado, representaciones de la realidad, comprender su existencia, desarrollar su esfera afectiva y dotar de funcionalidad mediante su concatenación con las esferas cognitiva y volitiva a la personalidad como función psicológica superior reguladora del comportamiento. 


\section{Referencias}

ASMOLOV, A. G. Concepto de personalidad en la teoría de la actividad.

Conferencia del Seminario Internacional de Psicología: Actualidad, aplicaciones y perspectivas de la teoría histórico-cultural; Puebla, México, 2002.

ASMOLOV, A. G. The Subject Matter of the Psychology of Personality, Soviet Psychology. v. 22, n. 4, p. 23-43, 2009. DOI:

https://doi.org/10.2753/RPO1061-0405220423.

BOLÍVAR, A. Educación para la ciudadanía: algo más que una asignatura. España: Graó, 2007.

BOURDIEU, P.; PASSERON, J. La reproducción: elementos para una teoría del sistema de enseñanza. México: Fontamara, 1996.

BOZHOVICH, L. I. La personalidad y su formación en la edad infantil. La Habana: Editorial Pueblo y Educación, 1976.

BOZHOVICH, L. I. The Struggle for Concrete Psychology and the Integrated Study of Personality. Journal of Russian \& East European Psychology, v. 47, n. 4, p.28-58, 2009. DOI: https://doi.org/10.2753/RPO1061-0405470402.

CASAANY, D. La cocina de la escritura. Barcelona: Anagrama, 1993.

DUSAVITSKII, A. K. On the Ideal Form of Personality Development in the System of Developmental Education. Journal of Russian \& East European Psychology, v. 41, n. 5, p.34-50, 2003. DOI: https://doi.org/10.2753/RPO1061-0405410534.

FANON, F. Los condenados de la tierra. México: Fondo de cultura económica, 1963.

FERREIRO, E. Alfabetización: teoría y práctica. México: Siglo Veintiuno Editores, 2002.

FREIRE, P. Pedagogía del oprimido. Uruguay: Siglo Veintiuno Editores, 1999.

FREIRE, P.; MACEDO, D. Alfabetización. Lectura de la palabra y lectura de la realidad. España: Paidós, 1989.

GALPERIN, P. Linguistic consciousness and some questions of the relationship between language and thought. Journal of Russian and East European Psychology. v. 30, n. 4, p. 81-91. 1992(a). DOI: https://doi.org/10.2753/RPO1061-0405300481.

GALPERIN, P. The Problem of Activity in Soviet Psychology. Journal of Russian and East European Psychology. v. 30, n. 4, p. 37-59. 1992(b). DOI: https://doi.org/10.2753/RPO1061-0405300437. 
GIMÉNEZ, G. Estudios sobre la cultura y las identidades sociales. México: Consejo Nacional para la Cultura y las Artes, 2007.

GONZÁLEZ, F. Psicología de la personalidad. La Habana: Editorial Pueblo y Educación, 1985.

GONZÁLEZ, F.; MITJÁNS, A. La personalidad su educación y desarrollo. La Habana, Cuba: Pueblo y Educación, 1999.

INSTITUTO NACIONAL PARA LA EDUCACIÓN DE LOS ADULTOS. 35 años creciendo con educación. México: Autor, 2016.

INSTITUTO NACIONAL PARA LA EDUCACIÓN DE LOS ADULTOS. A 30 años de su creación, semblanza de la labor del Instituto Nacional para la Educación de los Adultos. México: Autor, 2011.

JOLIBERT, J.; SRAÏKI, C. Niños que construyen su poder de leer y escribir. Buenos Aires: Manantial, 2009.

LEONTIEV, A. N. Actividad, Conciencia y Personalidad. Argentina, Buenos Aires: Ediciones Ciencias del Hombre, 1978.

LEONTIEV, A. N. El hombre y la cultura. México: Grijalbo, 1969.

LEONTIEV, A. N. The Genesis of Activity. Journal of Russian and East European Psychology. v. 43, n. 4, p. 58-71, 2005 (a). DOI: https://doi.org/10.1080/10610405.2005.11059253.

LEONTIEV, A.N. Will, Journal of Russian \& East European Psychology. v. 43, n. 4, 76-92, 2005 (b). DOI: https://doi.org/10.1080/10610405.2005.11059256.

LÓPEZ, E. (comp.). Interculturalidad, educación y ciudadanía: perspectivas latinoamericanas. Bolivia: Plural editores, 2009.

LURIA, A. R. Lenguaje y comportamiento. Madrid: Editorial Fundamentos, 1984.

LURIA, A. R. Higher Cortical Functions. New York: Appleton, 1995.

LURIA, A. R. The nature of human conflicts. USA: New York, 1976.

MALAGÓN, J. C. Sentidos y significados en torno a la identidad ciudadana en el proceso de alfabetización. Tesis para obtener el título de Licenciado en Psicología Educativa. UPN-Ajusco, 2020.

MARX, F. Le manifeste du Parti communiste. France: Union Générale D’Éditions, 1962. 
MOLTÓ, J. Psicología de las emociones entre la biología y la cultura. Valencia: Albatros Ediciones, 1995.

NEBYLÍTZIN, V. D. Las propiedades fundamentales del sistema nervioso humano como base neurofisiológica de la personalidad, en Smirnov, A. A., Luria, A. R.; Nebylítzin, V. D. Fundamentos de psicofisiología. España: Siglo XXI, pp. 299-340, 1983.

ORTIZ, G.; MARTÍNEZ, A. La funcionalidad de la personalidad a través de la orientación activa. Tesis para obtener el título de Licenciado en Psicología, UNAM, 2003.

ORTIZ, G., CRUZ, F.; LEURS, A. La cultura en psicología: de los objetos de estudio a la complejización representacional, en HERNÁNDEZ, S., MENDOZA, J. Y ORTIZ, G. Psicología, Cultura y Educación. México: UPN. pp. 17-44, 2017.

PASQUALINI, J. C; MARTINS, L. M. Dialética singular-particular-universal: implicações do método materialista dialético para a psicologia. Psicologia \& Sociedade, v. 27, n. 2, p. 361-370, 2015. DOI: https://doi.org/10.1590/180703102015v27n2p362.

PETROVSKY, A. Psicología evolutiva y pedagogía. Argentina: Cártago, 1985.

RUBINSTEIN, S. El ser y la conciencia. México: Grijalbo, 1963.

SHELDON, K., GORDEEVA, T., LEONTIEV, D., LYNCH, M., OSIN, E., RASSKAZOVA, E.; DEMENTY, L. Freedom and responsibilty together: personality, experimental, and cultural demonstrations. Journal of research personality, v. 73, p.63-74, 2018. DOI: https://doi.org/10.1016/j.jrp.2017.11.007.

SKLIAR, C. zy si el otro no estuviera ahî?: notas para una pedagogía (improbable) de la diferencia. Argentina: Miño y Dávila Editores, 2007.

SMIRNOV, A. A., RUBINSTEIN, S. L., LEONTIEV, A. N.; TIEPLOV B. M. Psicología. México, D. F: Grijalbo, 1997.

TEBEROSKY, A.; SOLER, M. Contextos de alfabetización inicial. Barcelona: Editorial Horsori, 2003.

TSVÉTKOVA, L. S. Reeducación del lenguaje, la lectura y la escritura. Barcelona: Fontanella, 1977.

VAN-MANEN, M. Investigación educativa y experiencia vivida. España: Idea books, 2003.

VYGOTSKY, L. S. Obras escogidas. España: Aprendizaje Visor, 1995. 
VYGOTSKY, L. S. Pensamiento y lenguaje. Cuba, La Habana: Pueblo y Educación, 1999.

VYGOTSKY, L. S. Teoría de las emociones. Estudio histórico-psicológico. España: Akal, 2004.

VYGOTSKY, L. S. La conciencia como problema de la psicología del comportamiento. Obras Escogidas Tomo I, s.f. a. https://vygotskitraducido.blogspot.com/2020/02/.

VYGOTSKY, L. S. La psique, la conciencia; el inconsciente. Obras Escogidas Tomo I, s.f. b. https://vygotski-traducido.blogspot.com/2020/02/.

ZEIGÁRNIK, B. V. Introducción a la patopsicología. España: Akal, 1981. 